\title{
Antitumor effects of the antiparasitic agent ivermectin Paper Antitumor effects of the antiparasitic agent ivermectin via inhibition of Yes-associated protein 1 expression in gastric cancer
}

\author{
Sho Nambara ${ }^{1}$, Takaaki Masuda ${ }^{1}$, Miki Nishio ${ }^{2,3}$, Shotaro Kuramitsu ${ }^{1}$, Taro Tobo ${ }^{4}$, \\ Yushi Ogawa ${ }^{5}$, Qingjiang $\mathrm{Hu}^{1}$, Tomohiro Iguchi ${ }^{6}$, Yousuke Kuroda ${ }^{1}$, Shuhei \\ Ito $^{1}$, Hidetoshi Eguchi ${ }^{1}$, Keishi Sugimachi', ${ }^{1,6}$ Hiroshi Saeki', Eiji Oki ${ }^{7}$, Yoshihiko \\ Maehara', Akira Suzuki ${ }^{2,3}$ and Koshi Mimori ${ }^{1}$ \\ ${ }^{1}$ Department of Surgery, Kyushu University Beppu Hospital, Beppu, Oita 874-0838, Japan \\ ${ }^{2}$ Medical Institute of Bioregulation, Graduate School of Medical Sciences, Kyushu University, Fukuoka 812-8582, Japan \\ ${ }^{3}$ Division of Molecular and Cellular Biology, Kobe University Graduate School of Medicine, Chuo-Ku, Kobe, Hyogo 650-0017, \\ Japan \\ ${ }^{4}$ Department of Pathology, Kyushu University Beppu Hospital, Beppu, Oita 874-0838, Japan \\ ${ }^{5}$ Digestive Disease Center, Showa University Northern Yokohama Hospital, Tsuzuki-Ku, Kanagawa 224-8503, Japan \\ ${ }^{6}$ Department of Gastroenterological Surgery, National Kyushu Cancer Center, Fukuoka 811-1395, Japan \\ ${ }^{7}$ Department of Surgery and Science, Graduate School of Medical Sciences, Kyushu University, Fukuoka 812-8582, Japan \\ Correspondence to: Koshi Mimori, email: kmimori@beppu.kyushu-u.ac.jp \\ Keywords: ivermectin; yes-associated protein 1 inhibitor; gastric cancer; antiproliferative effect; therapeutic target \\ Received: May 31, $2017 \quad$ Accepted: November 03, $2017 \quad$ Published: November 21, 2017
}

Copyright: Nambara et al. This is an open-access article distributed under the terms of the Creative Commons Attribution License 3.0 (CC BY 3.0), which permits unrestricted use, distribution, and reproduction in any medium, provided the original author and source are credited.

\section{ABSTRACT}

Yes-associated protein 1 (YAP1) acts as an oncogene through dephosphorylation and nuclear translocation, and nuclear accumulation of YAP1 is associated with poor prognosis in gastric cancer (GC). We previously identified ivermectin, an antiparasitic drug, as a YAP1 inhibitor. Here, we aimed to clarify whether ivermectin had antitumor effects on GC through inhibition of YAP1. First, we evaluated the antiproliferative effects of ivermectin on human GC cells using in vitro proliferation assays and a xenograft mouse model. YAP1-knockdown assays were performed to assess whether the sensitivity to ivermectin depended on YAP1 expression. Next, we explored the mechanism through which ivermectin regulated YAP1 expression or localization by immunoblotting and reverse transcription-quantitative polymerase chain reaction for YAP1 and the downstream gene CTGF. Finally, the clinical significance of YAP1 expression was examined using three independent GC datasets. We found that MKN1 GC cells were most sensitive to ivermectin, whereas MKN7 cells were most resistant. In MKN1 xenografts, ivermectin suppressed tumor growth, and the sensitivity of MKN1 cells to ivermectin was decreased by YAP1 knockdown. Ivermectin inhibited YAP1 nuclear expression and CTGF expression in MKN1 cells but not MKN7 cells. Moreover, ivermectin decreased YAP1 mRNA expression, thereby inhibiting nuclear accumulation of YAP1 in MKN1 cells. In survival analysis, low YAP1 mRNA expression was associated with a better prognosis in three independent GC datasets. In conclusion, we identified ivermectin as a potential antitumor agent and found a promising novel therapeutic strategy for inhibition of GC progression by blocking YAP1 expression. 


\section{INTRODUCTION}

Gastric cancer (GC) is the fifth most common malignancy and the third leading cause of cancer-related death worldwide [1]. Despite recent advances in medical treatments, such as chemotherapy and biological therapy, for the management of GC, patient survival remains poor, particularly for those with advanced disease [2], highlighting the need for the development of novel therapeutic agents.

Yes-associated protein 1 (YAP1) is upregulated and exhibits oncogenic properties in GC. Moreover, increased nuclear expression of YAP1 is associated with poor prognosis in patients with $\mathrm{GC}[3,4]$, colon cancer, ovarian cancer, and lung cancer [5-7]. YAP1 is a downstream target of the Hippo signaling pathway, which regulates organ size during development [8]. YAP1 acts as a transcriptional co-activator in the nucleus, activating TEA domain transcription factor (TEAD)-mediated transcription of cell proliferation genes, such as connective tissue growth factor (CTGF) [9]. Activation of the Hippo pathway phosphorylates YAP1 at Ser127, which inhibits the activity of YAP1 and results in retention of YAP1 in the cytoplasm $[10,11]$. Thus, nuclear YAP1 is a positive regulator of cell proliferation that is suppressed by Hippo signaling. Hence, inhibition of YAP1 expression may prevent tumor progression and improve prognosis in various malignancies, including GC [3-7].

We previously identified ivermectin as a potential YAP1 inhibitor by chemical compounds screening [12]. Ivermectin is a chemically modified derivative of avermectin, which was initially purified by Drs. Campbell and Omura as an effective antiparasitic agent $[13,14]$, earning these researchers a Nobel Prize in Physiology or Medicine in 2015. Recently, ivermectin has been reported to be a promising antitumor agent for various types of malignant tumors, including colon cancer, ovarian cancer, melanoma, and leukemia [15-18]. However, little is known about the molecular mechanisms underlying ivermectinmediated suppression of tumor growth, and no studies have evaluated whether ivermectin has antitumor effects in GC. Accordingly, such studies may enable ivermectin to be repositioned as a novel anticancer drug.

In this study, we aimed to clarify the antitumor effects of ivermectin in GC and evaluate the mechanisms through which regulation of YAP1 expression modulates these antitumor effects.

\section{RESULTS}

\section{Ivermectin suppressed GC growth in vitro and in a xenograft mouse model}

First, the antiproliferative effects of ivermectin on GC were examined by MTT assays and colony formation assays using GC cell lines. MTT assays demonstrated that the response to ivermectin was different among cell lines. Among the tested cell lines, we found that MKN1 cells were most sensitive to ivermectin $\left(\mathrm{IC}_{50}=10.2 \mu \mathrm{M}\right)$ and that $\mathrm{SH}$ 10-TC cells were also sensitive to the drug $\left(\mathrm{IC}_{50}=21.2 \mu \mathrm{M}\right.$; Figure 1A). Colony formation assays showed that ivermectin treatment significantly reduced colony formation in MKN1 cells (Figure 1B). In contrast, MKN7 cells were resistant to ivermectin $\left(\mathrm{IC}_{50}=31.9 \mu \mathrm{M}\right)$, as were MKN28 cells $\left(\mathrm{IC}_{50}=25.4 \mu \mathrm{M}\right.$; Figure 1A). Therefore, we defined MKN1 and SH-10-TC cells as ivermectin-sensitive cells and MKN7 and MKN28 cells as ivermectin-resistant cells. In addition, we performed apoptosis assays to determine whether ivermectin induced apoptosis in GC cells and found that ivermectin treatment did not induce apoptosis in vitro (Supplementary Figure 1A).

To evaluate the effects of ivermectin on GC cell growth in vivo, we employed a xenograft mouse model by injecting ivermectin-sensitive MKN1 cells subcutaneously into nude mice. As shown in Figure 1C, ivermectin treatment dramatically reduced tumor growth in mice. Macroscopically, ivermectin-treated tumors were much smaller than control tumors (Figure 1D). Consistent with these findings, tumor weight was reduced in ivermectintreated mice compared with that in the control group (Figure 1E). In addition, we also assessed whether mice showed any alterations in whole body weights after ivermectin treatment. The body weights of mice from the ivermectin-treated group were similar to those of control mice during the entire treatment period, except for 7 days after the start of treatment (Figure 1F). Finally, immunohistochemical analysis for xenografts tumor tissues showed that ivermectin-treated xenografts displayed weaker YAP1 and Ki67 staining than control mice (Figure 1G). Moreover, ivermectin treatment did not induce apoptosis in tumor tissues, as demonstrated by TUNEL assays (Supplementary Figure 1B). We attempted to establish a xenograft mouse model with subcutaneous tumors using SH-10-TC. However, we were unable to establish such a model. Taken together, these data suggested that ivermectin suppressed the growth of GC in vitro and in vivo by inhibiting proliferation rather than inducing apoptosis.

\section{The antiproliferative effects of ivermectin were dependent on YAP1 expression}

MTT assays were then conducted to investigate whether the proliferation of MKN1 cells (ivermectinsensitive) and MKN7 cells (ivermectin-resistant) was dependent on YAP1 expression. YAP1 knockdown induced significant downregulation of YAP1 protein expression in both MKN1 and MKN7 (Figure 2A). YAP1 knockdown significantly suppressed cell proliferation in MKN1 cells but not in MKN7 cells (Figure 2B). Thus, MKN1 cell proliferation was dependent on YAP1 expression, whereas MKN7 cell proliferation was independent of YAP1 expression. Next, we examined whether the effects of 
A
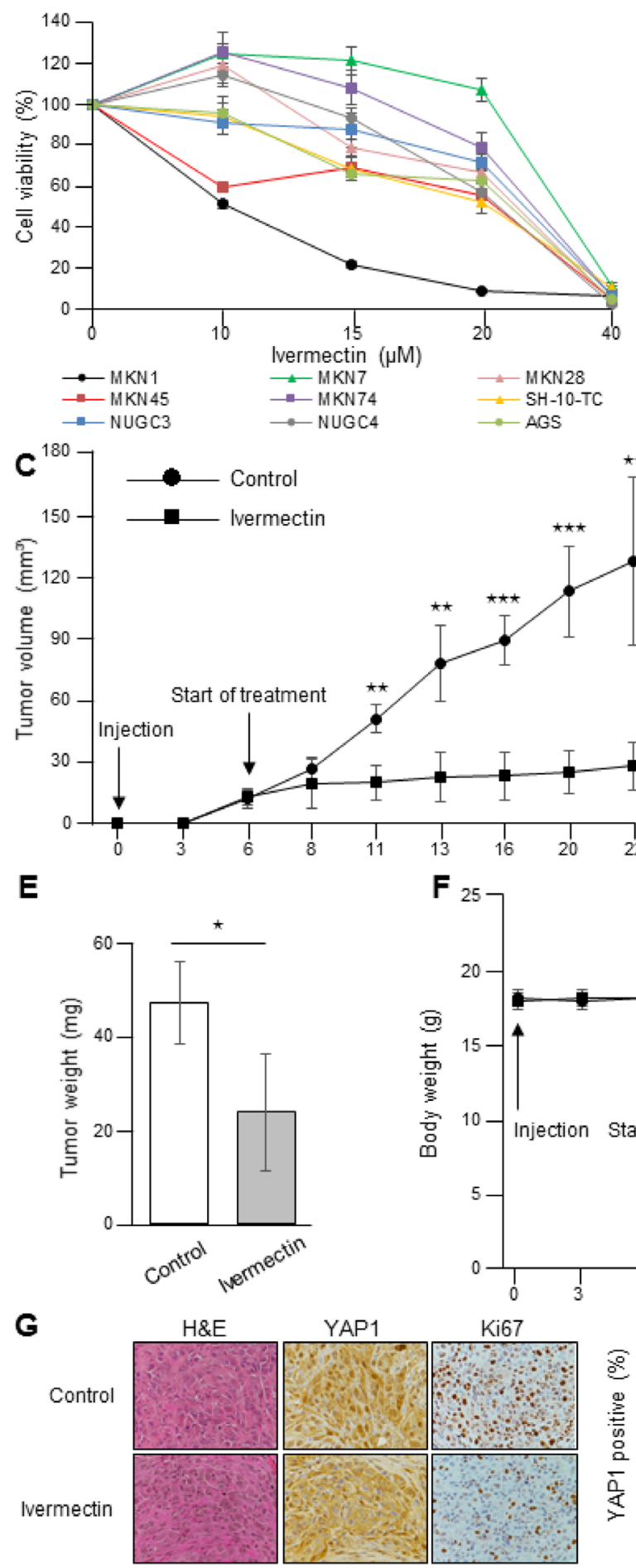

B

$$
\text { Ivermectin } \quad \begin{array}{rrrrr}
0 & 2.5 & 5 & 10 & (\mu \mathrm{M})
\end{array}
$$
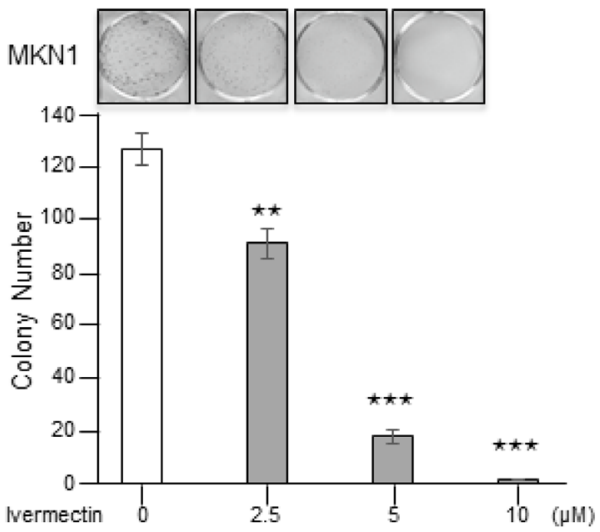

D

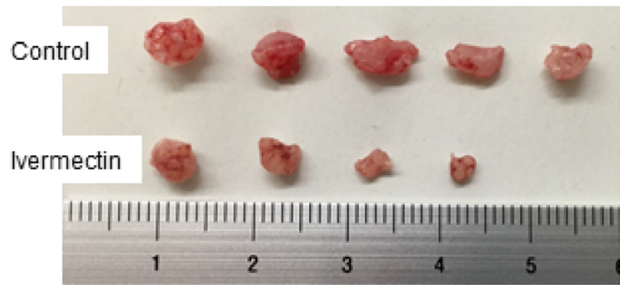

Days
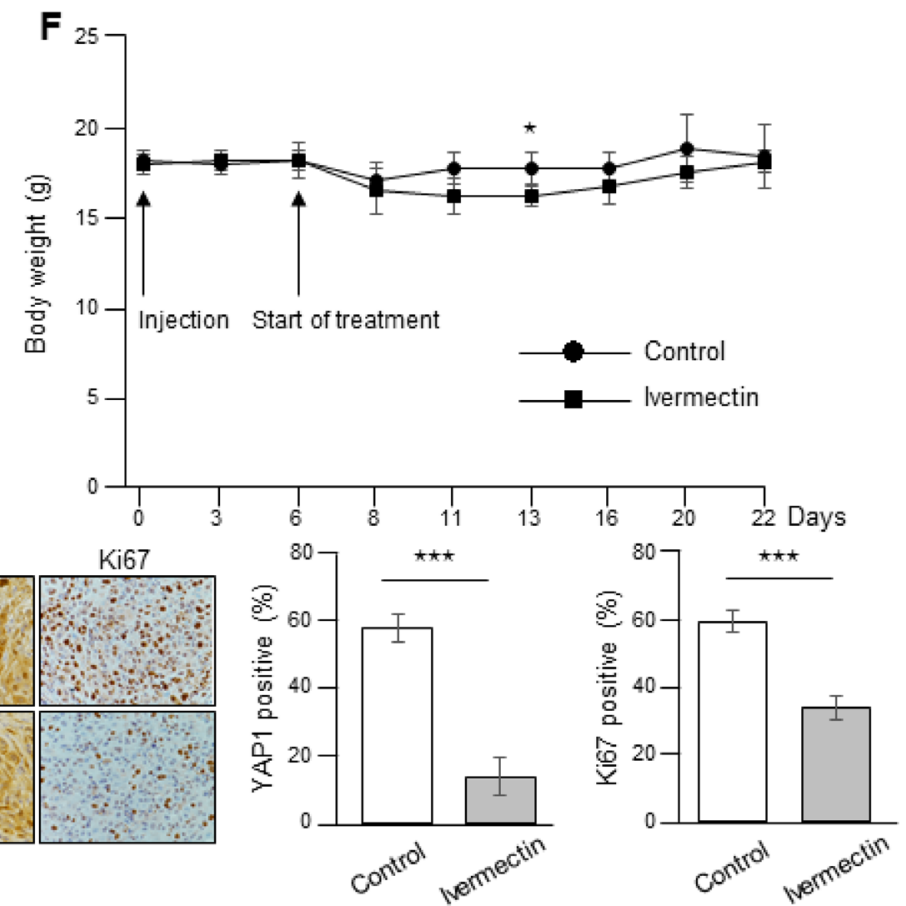

Figure 1: Ivermectin suppressed the growth of GC cells in vitro and in a xenograft mouse model. (A) Sensitivity of GC cells to ivermectin. Cell viability was measured by MTT assays in GC cells treated with the indicated concentrations of ivermectin for 48 h. (B) Top, Colony formation assays. Cells were cultured with the indicated concentrations of ivermectin for 10 days. Bottom, Total number of colonies. ${ }^{* *} P<0.005,{ }^{* * *} P<0.0005$. (C-G) Nude mice were inoculated with MKN1 cells and treated with ivermectin or control. (C) MKN1 tumor growth in mice treated with ivermectin or control. ${ }^{* *} P<0.005,{ }^{* * * *} P<0.0005$. (D) Subcutaneous tumors from control and ivermectin-treated mice. (E) Tumor weights at time of sacrifice. ${ }^{*} P<0.05$. (F) Body weights of control and ivermectin-treated mice during the entire experimental period (22 days). ${ }^{*} P<0.05$. (G) Left, Immunohistochemical staining for YAP1 and Ki67 in tumor tissues from control and ivermectin-treated mice. Original magnification, $\times 400$. Right, Data obtained by counting nuclear YAP1- and Ki67-positive tumor cells/tumor cells per fields. ${ }^{* * *} P<0.0005$. 
ivermectin on cell proliferation were dependent on YAP1 expression in YAP1-knockdown GC cells since ivermectin had previously been shown to inhibit YAP1 [12]. As shown in Figure 2C, the sensitivity of MKN1 cells to ivermectin was significantly decreased (siYAP1 $\mathrm{IC}_{50}=19.2 \mu \mathrm{M}$, siCTR $\left.\mathrm{IC}_{50}=13.9 \mu \mathrm{M}, \mathrm{RI}=1.4\right)$. This result was reproducible in another ivermectin-sensitive cell line, SH-10-TC (Supplementary Figure 2). In contrast, the sensitivity of MKN7 cells to ivermectin did not change

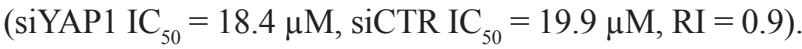

These data suggested that the antiproliferative effects of ivermectin depended on the expression level of YAP1 in GC cells.

\section{Ivermectin inhibited the nuclear accumulation of YAP1 in GC cells}

Next, we investigated whether ivermectin could regulate the nuclear accumulation of YAP1, which is important for its transcriptional functions, in GC cells. As

A

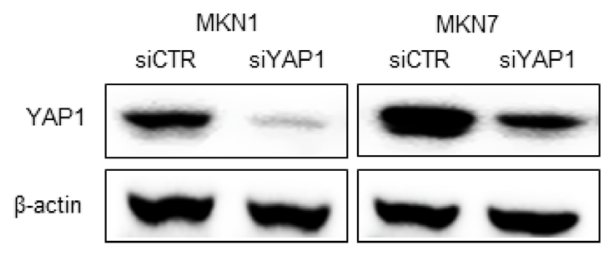

$\mathbf{B}$
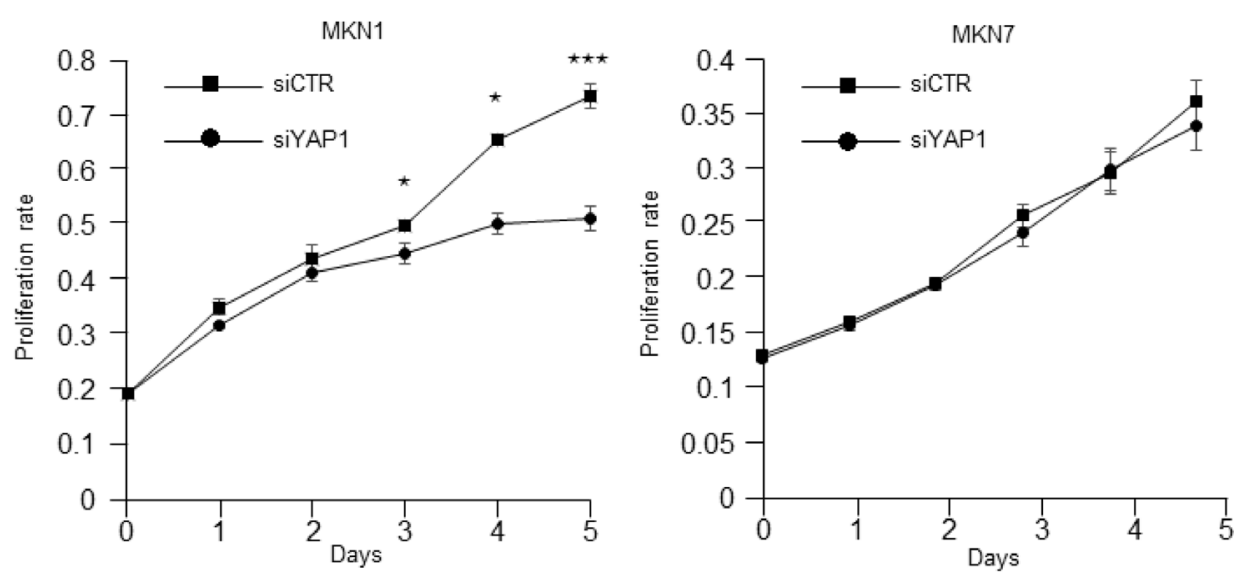

C
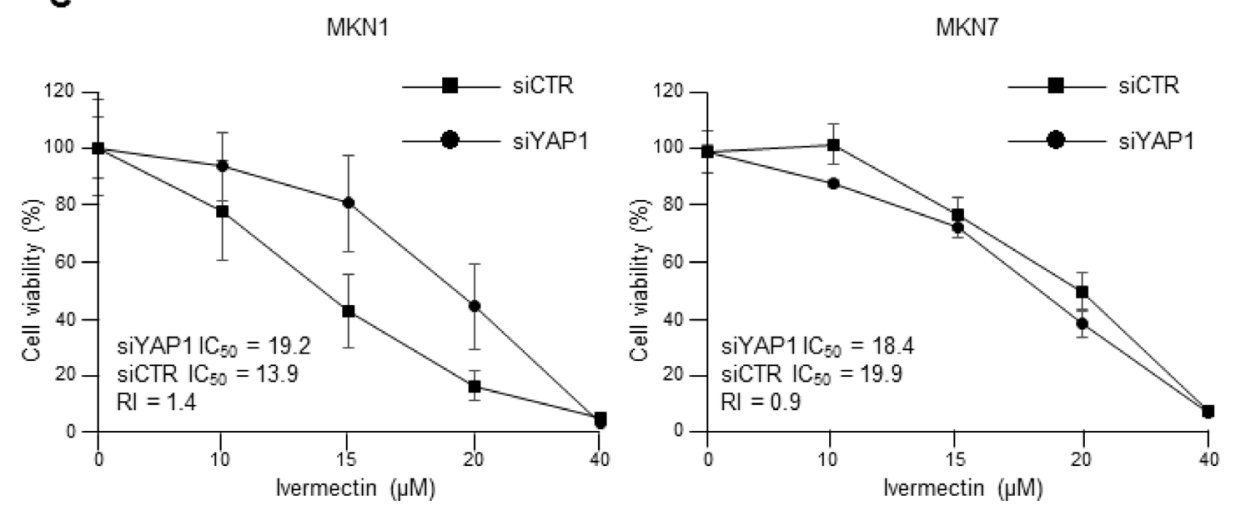

Figure 2: The antiproliferative effects of ivermectin were dependent on YAP1 expression. (A) Immunoblotting for total protein expression of YAP1 in YAP1 siRNA-transfected MKN1 and MKN7 cells and control siRNA-transfected cells. (B) MTT proliferation assays. The proliferation rates of YAP1 siRNA-transfected MKN1 and MKN7 cells were compared with that of control siRNA-transfected cells. ${ }^{*} P<0.05,{ }^{* * *} P<0.0005$. (C) IC $_{50}$ values and RIs for ivermectin in YAP1 siRNA-transfected MKN1 and MKN7 cells and control siRNA-transfected cells. RI; resistance index. 
shown in Figure 3A, ivermectin treatment decreased the nuclear expression of YAP1 and the YAP1 downstream target CTGF in MKN1 cells but not in MKN7 cells. Furthermore, ivermectin decreased nuclear accumulation of YAP1 of MKN1 cells in both a concentration- and timedependent manner (Figure 3B). Moreover, ivermectin did not induce the accumulation of phosphorylated YAP1 (Ser127; this phosphorylation event inhibits YAP1 activation) in the cytoplasm both in MKN1 and MKN7 cells (Figure 3C), suggesting that ivermectin did not inhibit canonical Hippo signaling. Immunofluorescence also showed that nuclear staining of YAP1 was markedly reduced after ivermectin treatment in MKN1 cells but not in MKN7 cells (Figure 3D). Thus, these results indicated that ivermectin suppressed the nuclear accumulation of YAP1 in ivermectin-sensitive GC cells.

\section{Ivermectin decreased YAP1 mRNA expression and reduced nuclear expression of YAP1}

Our experimental data showed that ivermectin suppressed the nuclear accumulation of YAP1 in GC cells. Therefore, we hypothesized that ivermectin may repress the expression of YAP1 mRNA, thereby leading to reduced levels of nuclear YAP1. Notably, ivermectin treatment significantly decreased YAP1 mRNA expression and whole and nuclear expression of YAP1 in MKN1 cells but not in MKN7 cells (Figure 3A and Figure 4A). This result was reproducible in other ivermectin-sensitive cells (i.e., SH-10-TC cells) and ivermectin-resistant cells (i.e., MKN28 cells; Figure 4B). Thus, ivermectin suppressed YAP1 protein expression by reducing YAP1 mRNA expression. In order to clarify the relationship between nuclear YAP1 protein expression and YAP1 mRNA levels, we performed immunoblotting and RTqPCR in 11 GC cell lines (Figure 4C). There was a positive but nonsignificant correlation between nuclear YAP1 protein expression and YAP1 mRNA levels, as demonstrated by Pearson's correlation coefficient (Supplementary Figure 3). However, there was a significant positive correlation by $\chi^{2}$ tests, as shown in Figure 4D, suggesting that nuclear expression of YAP1 protein was mildly correlated with YAP1 mRNA levels.

\section{Low expression of YAP1 mRNA in tumor tissues predicted good prognosis in patients with GC}

Because the positive correlation between nuclear expression of YAP1 protein and the expression of YAP1 mRNA suggested that YAP1 mRNA levels could be associated with tumor aggressiveness in GC, we assessed the clinical significance of YAP1 mRNA expression in GC. First, we examined the association between YAP1 mRNA expression and clinicopathological factors in the Kyushu dataset (Table 1). The low YAP1 mRNA expression group $(n=35)$ had a lower frequency of tissues showing poorly differentiated histology $(P<0.05)$, less tumor invasion
$(P<0.05)$, and lower rates of venous invasion $(P<0.05)$ compared with the high expression group $(n=66)$ in the Kyushu dataset. Next, we evaluated the survival rates in three independent datasets of GC. These analyses showed that low YAP1 mRNA expression was associated with a better prognosis than high $Y A P 1$ expression in all three GC datasets (Kyushu dataset: $P<0.05$, Singapore dataset: $P<0.0005$, Kaplan-Meier dataset: $P<0.0005$; Figure 5A). These clinical findings imply that downregulation of YAP1 mRNA could reduce the malignant characteristics of GC, supporting our experimental results.

\section{DISCUSSION}

This is the first study to assess the antitumor effects of ivermectin, a well-known antiparasitic drug, in GC. Notably, ivermectin suppressed the proliferation of GC cells in vitro by decreasing nuclear expression of YAP1 in a concentration- and time-dependent manner. Moreover, ivermectin exhibited strong antitumor effects in a xenograft mouse model, with almost no adverse effects. These findings indicated that ivermectin could be a promising therapeutic drug for YAP1-dependent GC.

Interestingly, our clinical analysis demonstrated that low expression of YAP1 mRNA in tumor tissues was associated with favorable clinicopathological phenotypes and good prognosis. These findings supported clinical evidence for a therapeutic strategy to inhibit YAP1 expression by ivermectin treatment in patients with GC. Furthermore, our findings suggested that high YAP1 mRNA expression may be a novel biomarker of poor prognosis and could be a surrogate marker for the therapeutic efficacy of ivermectin in GC.

In this study, we assessed the mechanism through which ivermectin regulates YAP1 function for the first time and demonstrated that ivermectin decreased YAP1 mRNA expression, resulting in reduced nuclear expression of YAP1 protein in GC cells (Figure 5B). Ivermectin-induced YAP1 mRNA downregulation was assumed to occur through transcriptional inactivation or mRNA destabilization [19]. Further experiments are required to clarify this mechanism.

In drug discovery, one successful strategy is the exploitation of established drugs that have already been approved for treatment of other diseases (i.e., drug repositioning or drug repurposing) [20, 21]. In this study, we showed that ivermectin, which is used as an antiparasitic drug and is commercially available at a low cost, could be an effective treatment option for patients with GC. Thus, we suggest that ivermectin may be repositioned as a novel drug for the treatment of YAP1-dependent GC.

In summary, ivermectin suppressed the growth of GC in vitro and in vivo by inhibiting YAP1 expression. Furthermore, GC with low YAP1 expression had favorable clinicopathological features and a good prognosis. These findings provided insights into the antiproliferative effects of ivermectin as a YAP1 inhibitor and established a 

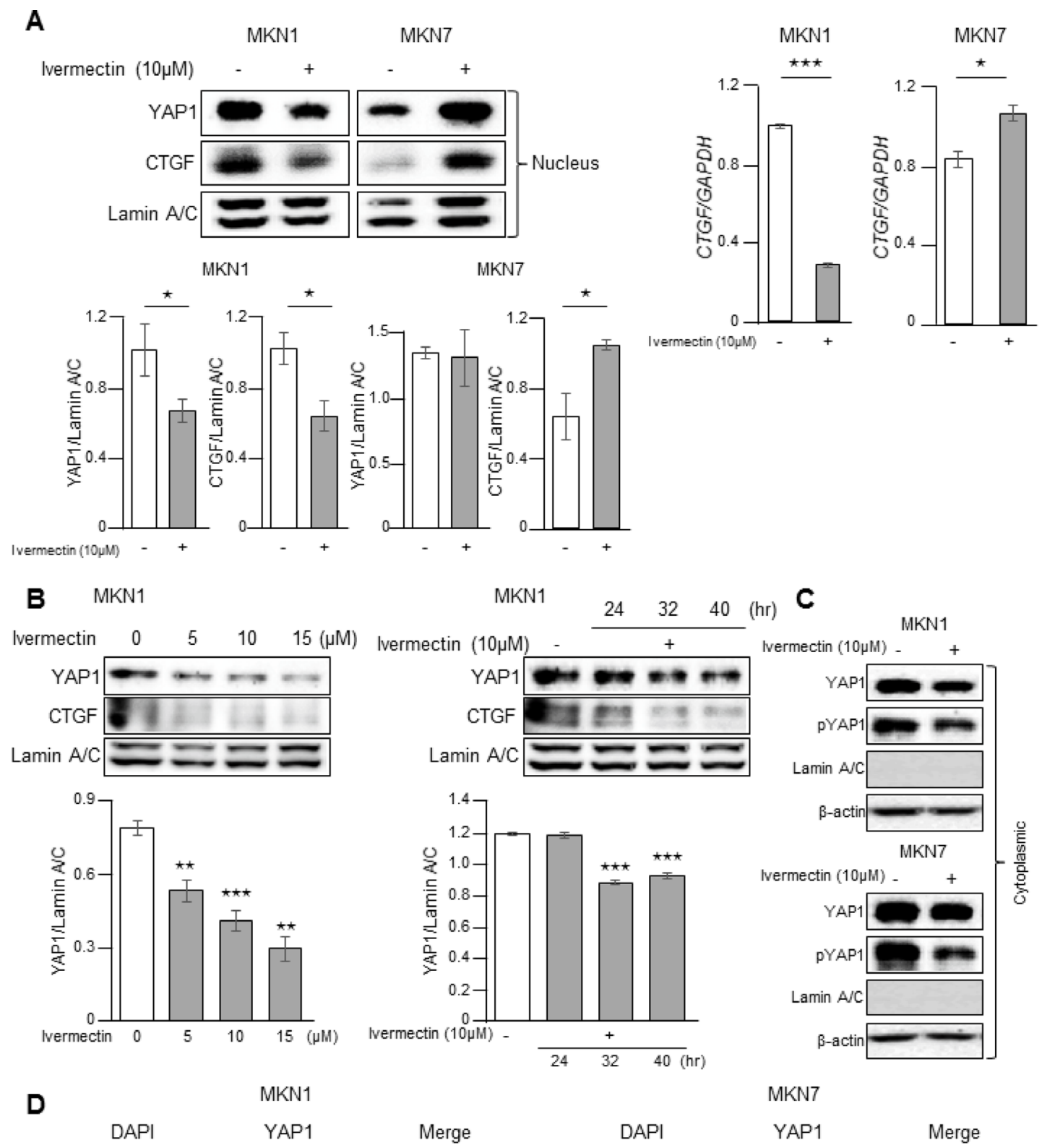


\section{A}
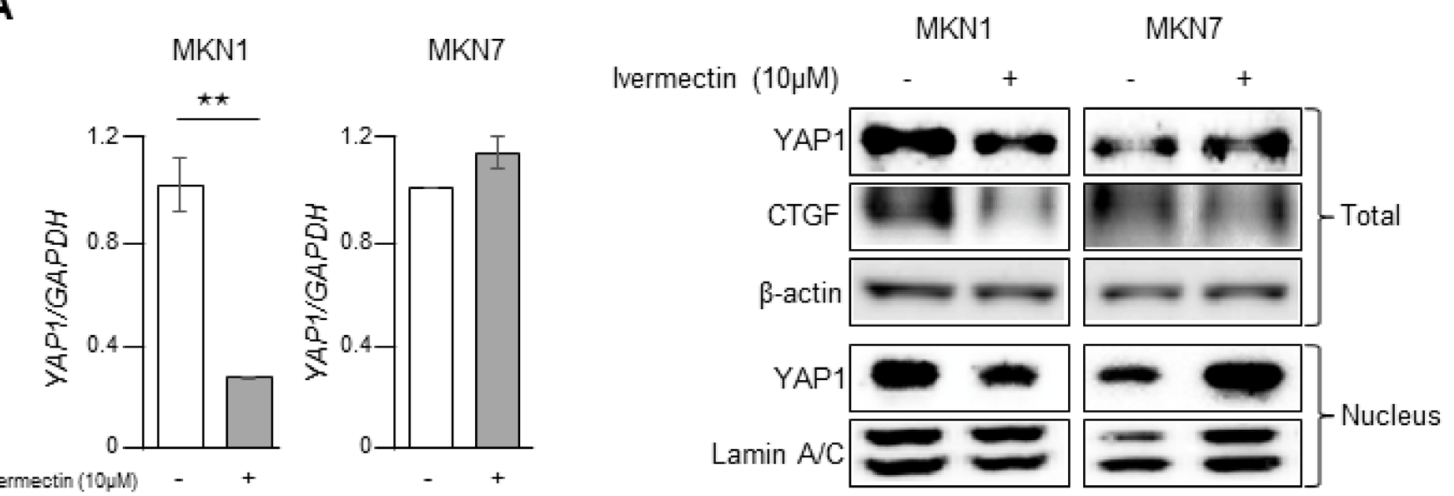

B
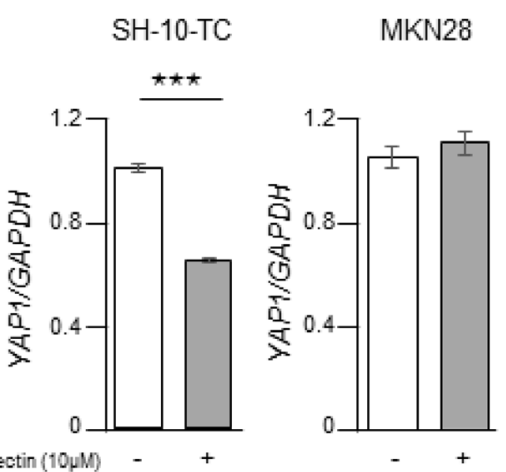

$\mathrm{MKN} 28$

$(10 \mu \mathrm{M})$

$\mathrm{SH}-10-\mathrm{TC}$

MKN28

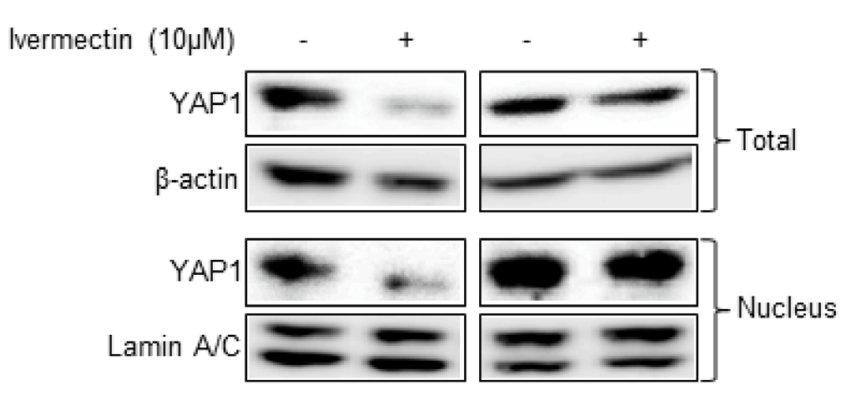

Ivermectin $(10 \mu M)$
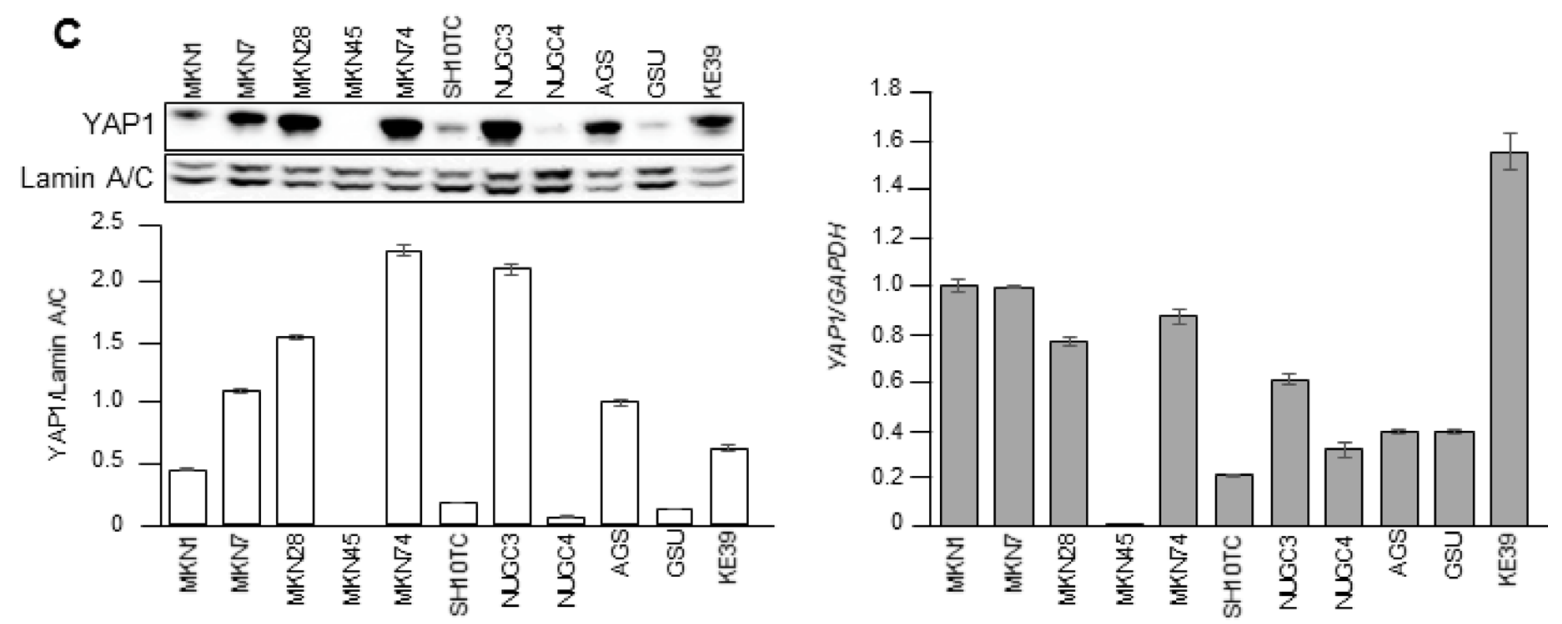

D

\begin{tabular}{ccccc}
\hline & & \multicolumn{2}{c}{ Intranuclear expression ofYAP1 } & \\
\cline { 3 - 4 } & & High & Low & $P$ - value \\
\hline \multirow{2}{*}{$\begin{array}{c}\text { YAP1 mRNA } \\
\text { expression }\end{array}$} & High & 5 & 1 & 0.036 \\
\hline
\end{tabular}

Figure 4: Ivermectin inhibited YAP1 expression by suppressing YAP1 mRNA levels in GC. (A) Left, RT-qPCR of YAP1 mRNA in MKN1 and MKN7 cells. ${ }^{* *} P<0.005$. Right, Immunoblotting for total and nuclear expression of YAP1 and CTGF in MKN1 and MKN7 cells. Cells were treated with vehicle (DMSO) or $10 \mu \mathrm{M}$ ivermectin for $24 \mathrm{~h}$. (B) Left, RT-qPCR of YAP1 mRNA in SH-10-TC and MKN28 cells. ${ }^{* * *} P<0.0005$. Right, Immunoblotting for total and nuclear expression of YAP1 and CTGF in SH-10-TC and MKN28 cells. Cells were treated with vehicle (DMSO) or $10 \mu \mathrm{M}$ ivermectin for $24 \mathrm{~h}$. (C) Left-top, Immunoblotting for nuclear expression of YAP1 in 11 GC cell lines. Left-bottom, Densitometry quantification of the band intensities. Right, RT-qPCR of YAP1 mRNA in 11 GC cell lines. (D) Relationship between nuclear expression of YAP1 and YAP1 mRNA expression in GC cells. 
Table 1: Correlation between YAP1 mRNA expression and clinicopathological factors of GC in the Kyushu dataset

\begin{tabular}{|c|c|c|c|}
\hline Factors & $\begin{array}{l}\text { Low }(n=35) \\
\text { Number }(\%)\end{array}$ & $\begin{array}{l}\text { High }(n=66) \\
\text { Number }(\%)\end{array}$ & $P$-value \\
\hline \multicolumn{4}{|l|}{ Age (years) } \\
\hline$<65$ & $16(45.7)$ & $31(47.0)$ & 0.904 \\
\hline$\geq 65$ & $19(54.3)$ & $35(53.0)$ & \\
\hline \multicolumn{4}{|l|}{ Sex } \\
\hline Male & $25(71.4)$ & $43(65.1)$ & 0.522 \\
\hline Female & $10(28.6)$ & $23(34.9)$ & \\
\hline \multicolumn{4}{|l|}{ Histology } \\
\hline well/moderate & $25(71.4)$ & $29(43.9)$ & $0.008^{*}$ \\
\hline poorly & $10(28.6)$ & $37(56.1)$ & \\
\hline \multicolumn{4}{|l|}{ Depth of invasion } \\
\hline$\leq \mathrm{SM}$ & $13(37.1)$ & $12(18.2)$ & $0.036^{*}$ \\
\hline$\geq \mathrm{MP}$ & $22(62.9)$ & $54(81.8)$ & \\
\hline \multicolumn{4}{|c|}{ Lymph node metastasis } \\
\hline Absent & $14(40.0)$ & $23(34.9)$ & 0.609 \\
\hline Present & $21(60.0)$ & $43(65.1)$ & \\
\hline \multicolumn{4}{|c|}{ Lymphatic invasion } \\
\hline Absent & $17(48.6)$ & $22(33.3)$ & 0.134 \\
\hline Present & $18(51.4)$ & $44(66.7)$ & \\
\hline \multicolumn{4}{|l|}{ Venous invasion } \\
\hline Absent & $30(85.7)$ & $43(65.1)$ & $0.028^{*}$ \\
\hline Present & $5(14.3)$ & $23(34.9)$ & \\
\hline \multicolumn{4}{|c|}{ Peritoneal metastasis } \\
\hline Absent & $31(88.6)$ & $56(84.8)$ & 0.606 \\
\hline Present & $4(11.4)$ & $10(15.2)$ & \\
\hline \multicolumn{4}{|l|}{ Liver metastasis } \\
\hline Absent & $33(94.3)$ & $61(92.4)$ & 0.726 \\
\hline Present & $2(5.7)$ & $5(7.6)$ & \\
\hline \multicolumn{4}{|l|}{ UICC TNM Stage } \\
\hline I, II & $22(62.9)$ & $36(54.6)$ & 0.421 \\
\hline III, IV & $13(37.1)$ & $30(45.4)$ & \\
\hline
\end{tabular}

SM: submucosa.

MP: muscularis propria.

UICC: Union for International Cancer Control.

TNM: tumor/node/metastasis.

${ }^{*} P<0.05$.

theoretical basis for preclinical evaluations of ivermectin for management of GC.

\section{MATERIALS AND METHODS}

\section{Cell culture}

The human GC cell lines MKN1, MKN7, MKN28, MKN45, MKN74, SH-10-TC, NUGC-3, NUGC-4, AGS, GSU, and KE-39 were purchased from RIKEN BioResource Center (Tsukuba, Japan). Cells were maintained in Roswell Park Memorial Institute (RPMI)
1640 medium containing $10 \%$ fetal bovine serum with $100 \mathrm{U} / \mathrm{mL}$ penicillin and $100 \mathrm{U} / \mathrm{mL}$ streptomycin sulfate and cultured in a humidified $5 \% \mathrm{CO}_{2}$ incubator at $37^{\circ} \mathrm{C}$.

\section{Total RNA extraction and reverse transcription- quantitative polymerase chain reaction (RT-qPCR)}

Total RNA from cell lines and tissues was extracted by the modified AGPC method using ISOGEN (Nippon Gene, Tokyo, Japan). RT was performed using $8 \mu \mathrm{g}$ of total RNA with M-MLV reverse transcriptase according to 
the manufacturer's instructions (Invitrogen, Carlsbad, CA, USA). qPCR assessments of YAP1, CTGF, and GAPDH were performed using LightCycler FastStart DNA Master SYBR Green I (Roche Diagnostics, Indianapolis, IN, USA) as previously described [22]. The expression levels of YAP1 and CTGF mRNA were normalized by $G A P D H$ mRNA as an internal control and are expressed as values relative to the expression level of the cDNA from Human Universal Reference Total RNA (Clontech, CA, USA). The primer sequences for qPCR were as follows: YAP1, forward 5'-CGCTCTTCAACGCCGTCA-3' and reverse 5'-AGTACTGGCCTGTCGGGAGT-3'; CTGF, forward 5'-TTGGCCCAGACCCAACTATG-3' and reverse 5'-CA GGAGGCGTTGTCATTGGT-3'; and GAPDH, forward, 5'-TTGGTATCGTGGAAGGACTCTA-3' and reverse, 5'-TGTCATATTTGGCAGGTT-3'.

\section{Protein extraction}

For total protein extraction, cells were lysed in lysis buffer (25 mM Tris-HCl [pH 7.5], $150 \mathrm{mM} \mathrm{NaCl,} 0.2 \mathrm{mM}$ EDTA, 0.1\% NP40, 5\% glycerol, and proteinase inhibitor cocktail). Extraction of nuclear and cytoplasmic protein was performed as previously described [23]. Briefly, the cell pellet was resuspended in buffer A (20 mM HEPES [pH 7.6], $10 \mathrm{mM} \mathrm{NaCl}, 1.5 \mathrm{mM} \mathrm{MgCl}, 0.2 \mathrm{mM}$ EDTA, $1 \mathrm{mM}$ DTT, $0.1 \%$ NP40, 20\% glycerol, and proteinase inhibitor cocktail), allowed to swell on ice for $10 \mathrm{~min}$, and then centrifuged to collect the cytoplasmic fraction (supernatant). The nuclear pellet was resuspended in buffer B (20 mM HEPES [pH 7.6], $500 \mathrm{mM} \mathrm{NaCl}, 1.5 \mathrm{mM}$ $\mathrm{MgCl}_{2}, 0.2 \mathrm{mM}$ EDTA, $1 \mathrm{mM}$ DTT, 0.1\% NP40, 20\% glycerol, and proteinase inhibitor cocktail), incubated for $30 \mathrm{~min}$ on ice, and centrifuged at 15,000 rpm for $15 \mathrm{~min}$ to collect the nuclear fraction.

\section{Immunohistochemistry}

Xenograft tumor tissues were fixed in 10\% formalin immediately after collection. Five-micron-thick sections were cut and stained with hematoxylin and eosin (H\&E), anti-YAP1 antibodies, and anti-Ki67 antibodies using the avidin-biotin-peroxidase method (LSAB2 kit; Dako, Kyoto, Japan). The primary antibodies against YAP1 and Ki67 were used at dilutions of 1:200 and 1:1000, respectively, and were purchased from Sigma-Aldrich (St. Louis, MO, USA)

A
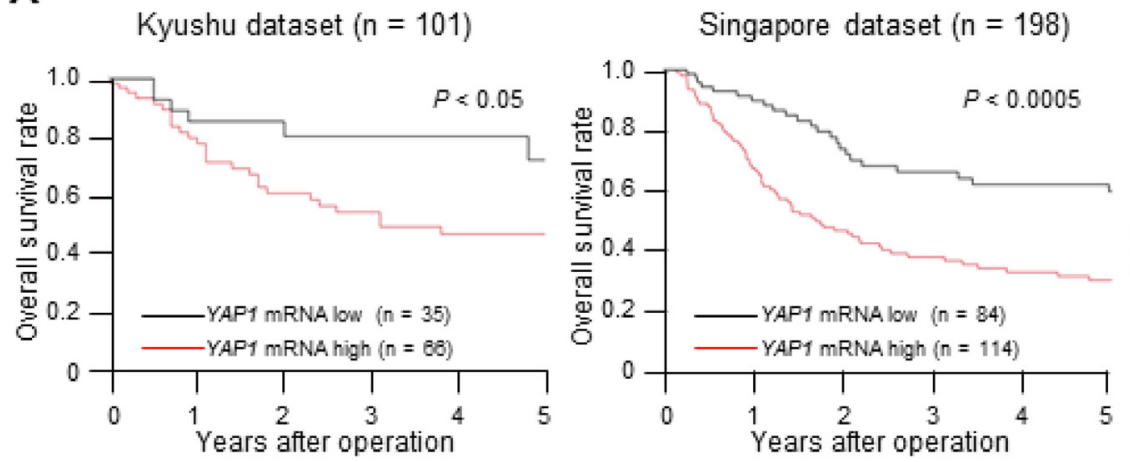

Kaplan-Meier dataset $(n=631)$
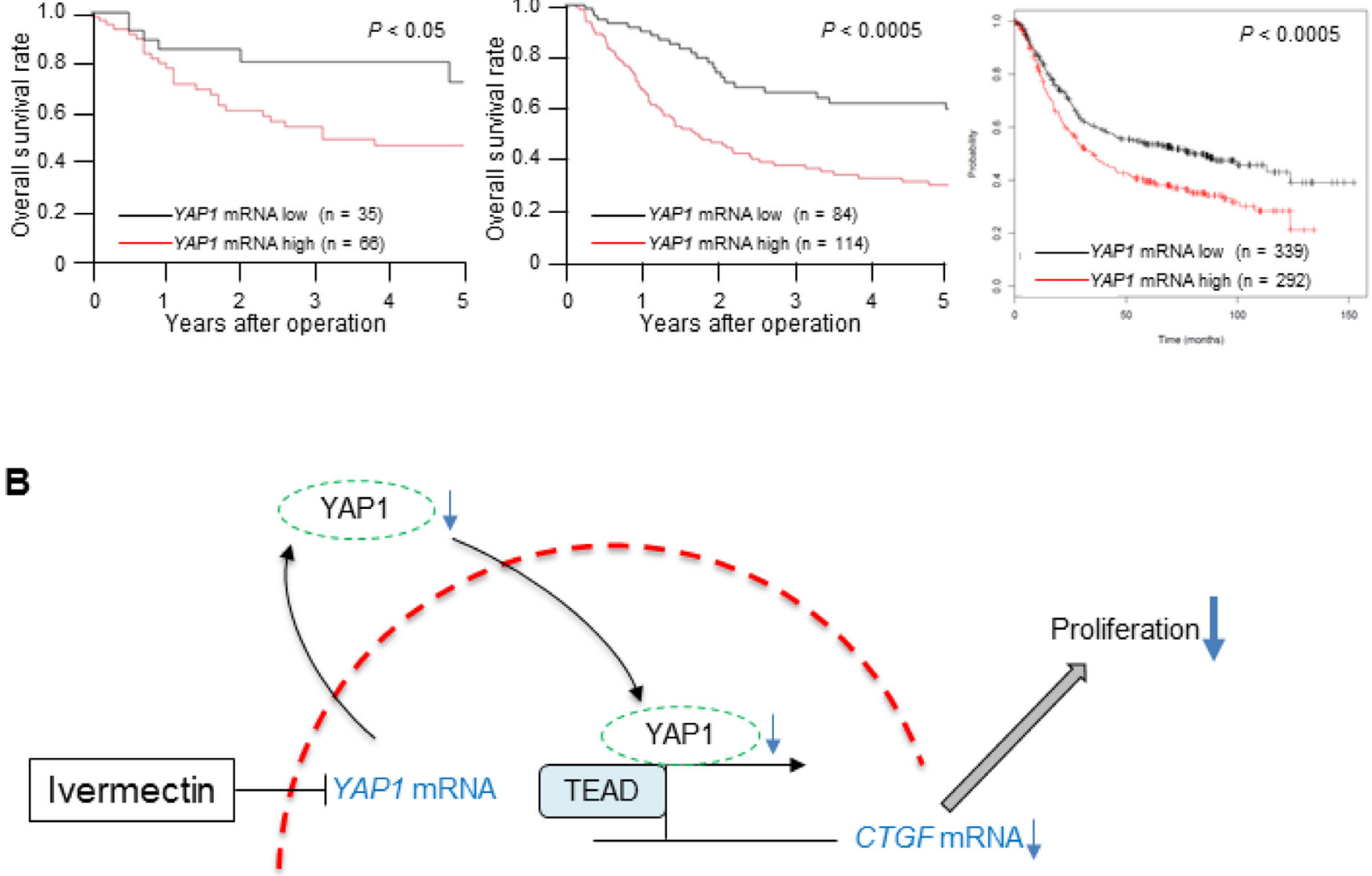

Figure 5: Low expression of YAP1 mRNA in tumor tissues predicted good prognosis in GC. (A) Overall survival rate of patients with GC according to YAPl mRNA expression in tumor tissues in three independent datasets (left: Kyushu dataset, middle: Singapore dataset, right: Kaplan-Meier dataset). (B) A proposed model showing how ivermectin inhibits YAP1 expression and suppresses tumor growth in GC. 
and Abcam (Cambridge, MA, USA), respectively. Tumor histology was independently reviewed by an experienced research pathologist at Kyushu University.

\section{Immunoblotting analysis}

Immunoblotting analysis was performed as previously described [24]. Briefly, equal amounts of protein (35 $\mu \mathrm{g}$ ) were electrophoresed on 4-20\% Tris-glycine gels and then electroblotted onto Immobilon-P Transfer Membranes (Merck Millipore, Billerica, MA, USA) at $70 \mathrm{~V}$ for $4 \mathrm{~h}$ at room temperature. Nonspecific binding sites were blocked with blocking buffer (Tris-buffered saline and $0.1 \%$ Tween-20 with 5\% nonfat milk powder) for $1 \mathrm{~h}$ at room temperature, and the blot was incubated with specific primary antibodies in blocking buffer (anti-YAP1, antiphospho-YAP1, anti-lamin $\mathrm{A} / \mathrm{C}$, and anti- $\beta$-actin antibodies at 1:1000 dilution; anti-CTGF antibodies at 1:200 dilution) at $4^{\circ} \mathrm{C}$ overnight. After washing, the blots were incubated with an appropriate secondary antibody conjugated with horseradish peroxidase for $1 \mathrm{~h}$ at room temperature. Blots were washed again, and detection was performed using an ImageQuant LAS 4000 Mini system (GE Healthcare Japan). Rabbit polyclonal antibodies targeting YAP1, phospho-YAP1, and lamin A/C were purchased from Cell Signaling Technology (Danvers, MA, USA) and goat polyclonal antibodies targeting CTGF and mouse monoclonal antibodies targeting $\beta$-actin were purchased from Santa Cruz Biotechnology (Santa Cruz, CA, USA). Immunoblotting densitometry analysis was performed using ImageJ software (NIH, Bethesda, MD, USA) [25]. The expression levels of YAP1 and CTGF protein were normalized according to the levels of lamin $\mathrm{A} / \mathrm{C}$ for nuclear extracts. Protein concentrations were quantified by Bradford protein assays.

\section{Immunofluorescence}

Fresh cells were fixed in 4\% paraformaldehyde for $15 \mathrm{~min}$, followed by permeabilization with $0.3 \%$ Triton $\mathrm{X} 100$. Cells were blocked with $3 \%$ bovine serum albumin for $1 \mathrm{~h}$ and incubated with primary antibodies at $4^{\circ} \mathrm{C}$ overnight. Cells were then washed with PBS, incubated with fluorescence-conjugated secondary antibodies for $1 \mathrm{~h}$, and stained with DAPI. Images were acquired using a fluorescent microscope (BZ-X700; KEYENCE, Tokyo, Japan). The primary antibody against YAP1, which was the same as that used in immunohistochemistry, was used at a dilution of 1:100. The secondary antibody, Alexa Fluor 555 Conjugate, was purchased from Cell Signaling Technology.

\section{YAP1 siRNA transfection}

YAP1-specific siRNA (Silencer Predesigned siRNA: sense, GGUGAUACUAUCAACCAAATT and antisense, UUUGGUUGAUAGUAUCACCTG) and negative control
siRNA (Silencer Negative Control 1 siRNA) were purchased from Ambion. Transfection of MKN1, SH10 -TC, and MKN7 cells $\left(1 \times 10^{4}\right.$ cells/well in 24-well plates) with siRNA oligonucleotides was performed using Lipofectamine RNAiMAX (Invitrogen) according to the manufacturer's instructions.

\section{MTT assay}

The short-term effects of ivermectin on GC cell growth and GC cell proliferation were assessed using 3-(4,5-dimethylthiazol-2-yl)-2,5-diphenyltetrazolium bromide (MTT) assays (Roche Applied Science), as previously described [26]. After incubation for $24 \mathrm{~h}$, followed by ivermectin treatment or siRNA transfection, the cells were cultured for an additional 0-120 h, and the absorbance of the samples was measured.

Resistance Index (RI) = half-maximal inhibitory concentration $\left(\mathrm{IC}_{50}\right)$ value of cells transfected with YAP1 siRNA/IC ${ }_{50}$ value of cells transfected with negative control siRNA.

\section{Colony formation assay}

The long-term effects of ivermectin on GC cell growth were assessed using colony formation assays. Cells were seeded at a density of 3000 cells/well in 6-well plates and treated with the indicated concentrations of ivermectin or vehicle control. After 10 days, the colonies were stained using a Differential Quik Stain Kit (Sysmex, Kobe, Japan) according to the manufacturer's instructions. Visible colonies were photographed using a Chemiluminescence Imaging FUSION SOLO S (VILBER, Marne la Vallée, France). Colony counts were determined using ImageJ software.

\section{Terminal deoxynucleotidyl transferase dUTP nick-end labeling (TUNEL) assay}

TUNEL staining of xenograft tumor tissues was performed using a kit according to the manufacturer's instructions (Wako Pure Chemical Industries, Osaka, Japan).

\section{Apoptosis assay}

Apoptosis was measured by immunoblotting analysis of cleaved poly (ADP-ribose) polymerase (PARP) and procaspase 3 using corresponding antibodies (Abcam, Cambridge, MA, USA) according to the manufacturer's instructions.

\section{Xenograft mouse model}

Five-week-old female BALB/c nu/nu mice were obtained from SLC, Inc. and maintained under specific pathogen-free conditions. All animal procedures were performed in compliance with the Guidelines for the 
Care and Use of Experimental Animals established by the Committee for Animal Experimentation of Kyushu University. For the xenograft model, $1 \times 10^{6} \mathrm{MKN} 1$ and SH-10-TC cells in $150 \mu \mathrm{L}$ serum-free medium were injected subcutaneously into the left flanks of the mice. After visual detection of tumors, mice were treated with cyclodextrin (45\%)-conjugated ivermectin (10 mg/kg, i.p., daily) or cyclodextrin carrier alone (control). Tumor sizes were measured every 3-4 days with a digital caliper and calculated using the following formula: tumor volume $=$ length $\times$ width $^{2} \times 0.5$. Mice were euthanized for analysis at 22 days after injection.

\section{Patients with GC and collection of clinical samples}

Primary GC samples were obtained from 101 patients who underwent surgery at Kyushu University Beppu Hospital and affiliated hospitals from 1992 to 2009 (Kyushu dataset). All patients had a histological diagnosis of GC and were followed at 3-month intervals. The median follow-up period was 2.2 years. All patients were treated in accordance with the Japanese gastric cancer treatment guidelines edited by the Japanese Gastric Cancer Association. Written informed consent was obtained from all patients, and the Institutional Review Board of our university approved this study. Sample collection was performed as previously described [24]. Data on patient age, sex, histology, tumor depth of invasion, lymph node metastasis, lymphatic invasion, venous invasion, peritoneal metastasis, liver metastasis, and clinical stage were obtained from clinical and pathological records.

\section{Singapore dataset analysis}

We obtained YAP1 mRNA expression and survival data for 198 available GC cases from the Singapore dataset, as previously described [27]. Gene expression array data were deposited in the Gene Expression Omnibus database under accession number GSE30601.

\section{Kaplan-meier plotter analysis}

The Kaplan-Meier plotter (www.kmplot.com), an online database that includes gene expression and clinical data, was used to generate the Kaplan-Meier overall survival plot as previously described (Kaplan-Meier dataset) [28].

\section{Statistical analysis}

For continuous variables, data were expressed as means \pm standard deviations, and statistical analyses were performed using Student's $t$ tests. Categorical variables were compared using Pearson's correlation coefficients and $\chi^{2}$ tests or Fisher's exact tests. Overall survival was estimated using the Kaplan-Meier method, and survival curves were compared using log-rank tests. Based on the levels of YAP1 mRNA expression in the Kyushu and Singapore datasets, cases were divided into two groups by the minimum $P$-value approach, a comprehensive method to find the optimal risk separation cutoff point in continuous gene expression measurement [29]. For YAP1 expression analysis in GC cells, we divided the 11 GC cells into two groups based on the median YAP1 expression. All tests were analyzed by JMP 12 software (SAS Institute, Cary, NC, USA). Clinicopathological factors and clinical stage were classified using the tumornode-metastasis (TNM) system of classification.

\section{ACKNOWLEDGMENTS}

We thank M. Oshiumi, M. Utou, K. Oda, M. Kasagi, S. Sakuma, N. Mishima, and T. Kawano for their excellent technical assistance.

\section{CONFLICTS OF INTEREST}

The authors declare no conflicts of interest.

\section{FUNDING}

This work was supported in part by the following grants and foundations: Japan Society for the Promotion of Science (JSPS) Grant-in-Aid for Science Research (grant nos. 16K07177, 16K10543, 16K10397, 16K19197, 16K19107, 16H01576, 26461980, and 26293303); OITA Cancer Research Foundation; Eli Lilly Japan K.K Grant 2017; Daiwa Securities Health Foundation.

\section{REFERENCES}

1. Ferlay J, Soerjomataram I, Dikshit R, Eser S, Mathers C, Rebelo M, Parkin DM, Forman D, Bray F. Cancer incidence and mortality worldwide:sources, methods and major patterns in GLOBOCAN 2012. Int J Cancer. 2015; 136:E359-86.

2. DeSantis CE, Lin CC, Mariotto AB, Siegel RL, Stein KD, Kramer JL, Alteri R, Robbins AS, Jemal A. Cancer treatment and survivorship statistics, 2014. CA Cancer J Clin. 2014; 64:252-71.

3. Kang W, Tong JH, Chan AW, Lee TL, Lung RW, Leung PP, So KK, Wu K, Fan D, Yu J, Sung JJ, To KF. Yes-associated protein 1 exhibits oncogenic property in gastric cancer and its nuclear accumulation associates with poor prognosis. Clin Cancer Res. 2011; 17:2130-9.

4. Sun D, Li X, He Y, Li W, Wang Y, Wang H, Jiang S, Xin Y. YAP1 enhances cell proliferation, migration, and invasion of gastric cancer in vitro and in vivo. Oncotarget. 2016; 7:81062-76. https://doi.org/10.18632/oncotarget.13188.

5. Lee KW, Lee SS, Kim SB, Sohn BH, Lee HS, Jang HJ, Park YY, Kopetz S, Kim SS, Oh SC, Lee JS. Significant association of oncogene YAP1 with poor prognosis and 
cetuximab resistance in colorectal cancer patients. Clin Cancer Res. 2015; 21:357-64.

6. Xia Y, Chang T, Wang Y, Liu Y, Li W, Li M, Fan HY. YAP promotes ovarian cancer cell tumorigenesis and is indicative of a poor prognosis for ovarian cancer patients. PLoS One. 2014; 9:e91770.

7. Kim MH, Kim YK, Shin DH, Lee HJ, Shin N, Kim A, Lee JH, Choi KU, Kim JY, Lee CH, Sol MY. Yes associated protein is a poor prognostic factor in well-differentiated lung adenocarcinoma. Int J Clin Exp Pathol. 2015; 8:15933-9.

8. Schlegelmilch K, Mohseni M, Kirak O, Pruszak J, Rodriguez JR, Zhou D, Kreger BT, Vasioukhin V, Avruch J, Brummelkamp TR, Camargo FD. Yap1 acts downstream of alpha-catenin to control epidermal proliferation. Cell. 2011; 144:782-95.

9. Zhao B, Ye X, Yu J, Li L, Li W, Li S, Yu J, Lin JD, Wang CY, Chinnaiyan AM, Lai ZC, Guan KL. TEAD mediates YAP-dependent gene induction and growth control. Genes Dev. 2008; 22:1962-71.

10. Kanai F, Marignani PA, Sarbassova D, Yagi R, Hall RA, Donowitz M, Hisaminato A, Fujiwara T, Ito Y, Cantley LC, Yaffe MB. TAZ:a novel transcriptional co-activator regulated by interactions with 14-3-3 and PDZ domain proteins. Embo j. 2000; 19:6778-91.

11. Zhao B, Li L, Tumaneng K, Wang CY, Guan KL. A coordinated phosphorylation by Lats and $\mathrm{CK} 1$ regulates YAP stability through SCF(beta-TRCP). Genes Dev. 2010; 24:72-85.

12. Nishio M, Sugimachi K, Goto H, Wang J, Morikawa T, Miyachi Y, Takano Y, Hikasa H, Itoh T, Suzuki SO, Kurihara H, Aishima S, Leask A, et al. Dysregulated YAP1/ TAZ and TGF-beta signaling mediate hepatocarcinogenesis in Mob1a/1b-deficient mice. Proc Natl Acad Sci U S A. 2016; 113:E71-80.

13. Ottesen EA, Campbell WC. Ivermectin in human medicine. J Antimicrob Chemother. 1994; 34:195-203.

14. Ikeda H, Omura S. Avermectin Biosynthesis. Chem Rev. 1997; 97:2591-610.

15. Melotti A, Mas C, Kuciak M, Lorente-Trigos A, Borges I, Ruiz i Altaba A. The river blindness drug Ivermectin and related macrocyclic lactones inhibit WNT-TCF pathway responses in human cancer. EMBO Mol Med. 2014; 6:1263-78.

16. Hashimoto H, Messerli SM, Sudo T, Maruta H. Ivermectin inactivates the kinase PAK1 and blocks the PAK1dependent growth of human ovarian cancer and NF2 tumor cell lines. Drug Discov Ther. 2009; 3:243-6.

17. Drinyaev VA, Mosin VA, Kruglyak EB, Novik TS, Sterlina TS, Ermakova NV, Kublik LN, Levitman M, Shaposhnikova VV, Korystov YN. Antitumor effect of avermectins. Eur J Pharmacol. 2004; 501:19-23.

18. Sharmeen S, Skrtic M, Sukhai MA, Hurren R, Gronda M, Wang X, Fonseca SB, Sun H, Wood TE, Ward R, Minden MD,
Batey RA, Datti A, et al. The antiparasitic agent ivermectin induces chloride-dependent membrane hyperpolarization and cell death in leukemia cells. Blood. 2010; 116:3593-603.

19. Wang H, Huang C, Zhao L, Zhang H, Yang JM, Luo P, Zhan BX, Pan Q, Li J, Wang BL. Histone deacetylase inhibitors regulate $\mathrm{P}$-gp expression in colorectal cancer via transcriptional activation and mRNA stabilization. Oncotarget. 2016; 7:49848-58. https://doi.org/10.18632/ oncotarget.10488.

20. Duenas-Gonzalez A, Garcia-Lopez P, Herrera LA, MedinaFranco JL, Gonzalez-Fierro A, Candelaria M. The prince and the pauper. A tale of anticancer targeted agents. Mol Cancer. 2008; 7:82.

21. Li YY, Jones SJ. Drug repositioning for personalized medicine. Genome Med. 2012; 4:27.

22. Masuda TA, Inoue H, Nishida K, Sonoda H, Yoshikawa Y, Kakeji Y, Utsunomiya T, Mori M. Cyclin-dependent kinase 1 gene expression is associated with poor prognosis in gastric carcinoma. Clin Cancer Res. 2003; 9:5693-8.

23. Dignam JD, Lebovitz RM, Roeder RG. Accurate transcription initiation by RNA polymerase II in a soluble extract from isolated mammalian nuclei. Nucleic Acids Res. $1983 ; 11: 1475-89$.

24. Hirata H, Sugimachi K, Komatsu H, Ueda M, Masuda T, Uchi R, Sakimura S, Nambara S, Saito T, Shinden Y, Iguchi T, Eguchi H, Ito $\mathrm{S}$, et al. Decreased Expression of Fructose-1,6-bisphosphatase Associates with Glucose Metabolism and Tumor Progression in Hepatocellular Carcinoma. Cancer Res. 2016; 76:3265-76.

25. Schneider CA, Rasband WS, Eliceiri KW. NIH Image to ImageJ:25 years of image analysis. Nat Methods. 2012; 9:671-5.

26. Liu R, Li J, Zhang T, Zou L, Chen Y, Wang K, Lei Y, Yuan K, Li Y, Lan J, Cheng L, Xie N, Xiang R, et al. Itraconazole suppresses the growth of glioblastoma through induction of autophagy:involvement of abnormal cholesterol trafficking. Autophagy. 2014; 10:1241-55.

27. Kurashige J, Hasegawa T, Niida A, Sugimachi K, Deng N, Mima K, Uchi R, Sawada G, Takahashi Y, Eguchi H, Inomata $\mathrm{M}$, Kitano $\mathrm{S}$, Fukagawa $\mathrm{T}$, et al. Integrated Molecular Profiling of Human Gastric Cancer Identifies DDR2 as a Potential Regulator of Peritoneal Dissemination. Sci Rep. 2016; 6:22371.

28. Shen JX, Liu J, Li GW, Huang YT, Wu HT. Mining distinct aldehyde dehydrogenase 1 (ALDH1) isoenzymes in gastric cancer. Oncotarget. 2016; 7:25340-9. https://doi. org/10.18632/oncotarget.8294.

29. Mizuno H, Kitada K, Nakai K, Sarai A. PrognoScan:a new database for meta-analysis of the prognostic value of genes. BMC Med Genomics. 2009; 2:18. 